\title{
Lens implementation on the GATE Monte Carlo toolkit for optical imaging simulation
}

\author{
Han Gyu Kang \\ Seong Hyun Song \\ Young Been Han \\ Kyeong Min Kim \\ Seong Jong Hong
}




\title{
Lens implementation on the GATE Monte Carlo toolkit for optical imaging simulation
}

\author{
Han Gyu Kang, ${ }^{a}$ Seong Hyun Song, ${ }^{a}$ Young Been Han, ${ }^{a}$ Kyeong Min Kim, ${ }^{b}$ and Seong Jong Hong ${ }^{a, c, \star}$ \\ ${ }^{a}$ Eulji University, Department of Senior Healthcare, Daejeon, Republic of Korea \\ ${ }^{b}$ Korea Institute of Radiological and Medical Science, Division of Medical Radiation Equipment, Nowon-gu, Seoul, Republic of Korea \\ 'Eulji University, Department of Radiological Science, Seongnam-si, Gyeonggi-do, Republic of Korea
}

\begin{abstract}
Optical imaging techniques are widely used for in vivo preclinical studies, and it is well known that the Geant4 Application for Emission Tomography (GATE) can be employed for the Monte Carlo (MC) modeling of light transport inside heterogeneous tissues. However, the GATE MC toolkit is limited in that it does not yet include optical lens implementation, even though this is required for a more realistic optical imaging simulation. We describe our implementation of a biconvex lens into the GATE MC toolkit to improve both the sensitivity and spatial resolution for optical imaging simulation. The lens implemented into the GATE was validated against the ZEMAX optical simulation using an US air force 1951 resolution target. The ray diagrams and the chargecoupled device images of the GATE optical simulation agreed with the ZEMAX optical simulation results. In conclusion, the use of a lens on the GATE optical simulation could improve the image quality of bioluminescence and fluorescence significantly as compared with pinhole optics. ๑ 2018 Society of Photo-Optical Instrumentation Engineers (SPIE) [DOI: 10.1117/1.JBO.23.2.026003]
\end{abstract}

Keywords: GATE optical imaging simulation; lens; bioluminescence imaging; fluorescence imaging.

Paper 170606R received Sep. 17, 2017; accepted for publication Jan. 22, 2018; published online Feb. 14, 2018.

\section{Introduction}

Optical imaging techniques, such as bioluminescence, fluorescence imaging, and Cerenkov luminescence imaging, are widely used in preclinical studies. ${ }^{1-3}$ At the same time, Monte Carlo (MC)-based optical simulations, such as the MC modeling of light transport in multilayered tissues (MCML), ${ }^{4}$ and the mouse optical simulation environment, ${ }^{5}$ have been proposed to model light transport inside tissues, such as that inside the brains of mice ${ }^{6}$ or humans. ${ }^{7}$ The Geant 4 Application for Emission Tomography (GATE) has been widely used to optimize various medical imaging modalities, including positron emission tomography, single-photon emission tomography, and computed tomography. ${ }^{8,9}$ Recently, the potential for optical imaging simulations using GATE was demonstrated by validating the optical scattering and absorption lengths versus an established MC optical simulation software package called $\mathrm{MCML}^{4}$ by Vesna Cuplov et al. ${ }^{10}$ The GATE can also be used for the simulation of nanoparticle-mediated near-infrared (NIR) thermal therapy. ${ }^{11}$ However, an optical lens, which is an essential component for optical imaging, has not yet been implemented into the GATE optical simulation toolkit. The aim of this study is to implement a lens into the GATE MC toolkit to improve both the sensitivity and spatial resolution for optical imaging simulation. In this study, the results of a GATE optical simulation, such as the images of a point spread function (PSF) and an US air force (USAF) 1951 resolution target, were compared with those of a ZEMAX nonsequential optical simulation. The bioluminescence imaging was simulated using GATE with various tissue materials such as epidermis and hypodermis to investigate the effect of optical phantom on image quality. The fluorescence

*Address all correspondence to: Seong Jong Hong, E-mail: hongseongj@eulji. ac.kr imaging was simulated with an indocyanine green (ICG) NIR fluorophore. The USAF 1951 resolution target imaging results with a biconvex lens were compared with those of pinhole optics to demonstrate the advantages of the lens over the pinhole.

\section{Materials and Methods}

\subsection{Lens Design}

The lens maker's equation was used to design a biconvex lens with a focal length of $7.5 \mathrm{~mm}^{12}$

$\frac{1}{f}=(n-1)\left[\frac{1}{R_{1}}-\frac{1}{R_{2}}+\frac{(n-1) d}{n R_{1} R_{2}}\right]$,

where $f$ is the focal length of the lens, $n$ is the refractive index of the lens material, $R_{1}$ and $R_{2}$ are the radii of curvature of the lens surface closest to the light source and farthest from the light source, respectively, and $d$ is the thickness of the lens (i.e., the distance between the two surface vertices along the principal axis). A ray diagram of the biconvex lens is shown in Fig. 1.

The object distance $S$ and image distance $S^{\prime}$ were calculated using the thin lens formula

$\frac{1}{S}+\frac{1}{S^{\prime}}=\frac{1}{f}$.

The image distance $S^{\prime}$ can be derived from Eq. (2) as follows:

$S^{\prime}=\frac{S \cdot f}{S-f}$

$1083-3668 / 2018 / \$ 25.00$ @ 2018 SPIE 


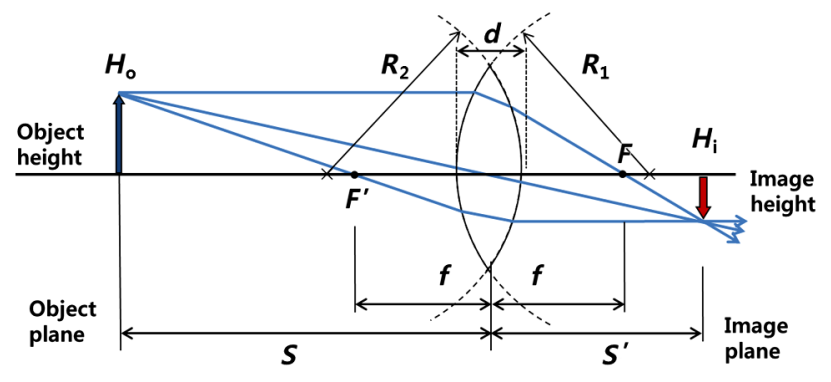

Fig. 1 Ray diagram of a biconvex lens.

Since we set the object distance to $30 \mathrm{~mm}$, the calculated image distance was, therefore, $10.01 \mathrm{~mm}$. The magnification factor $m$ of the lens can be determined based on the ratio of the image distance $S^{\prime}$ to the object distance $S$

$m=-\frac{S^{\prime}}{S}$

In this case, the magnification factor of the lens is -0.33 . The negative sign indicates that the image is flipped in both the horizontal and vertical directions on the image plane.

\subsection{Implementation of a Lens into GATE}

The lens design was implemented in a GATE V6.2 optical simulation. The lens consists of two spherical geometries, as shown in Fig. 2(a). The first spherical geometry (blue) is located at the left side of the lens and has an outer radius of $7.3 \mathrm{~mm}$, inner diameter of $6.3 \mathrm{~mm}$, and angle delta theta $\Delta \theta$ of $36 \mathrm{deg}$, respectively, as shown in Fig. 2(b). The second spherical geometry (purple) is located at the right side of the lens and has the same dimensions as the first spherical geometry, except for the reversed orientation along the principal axis ( $Z$-axis), as shown in Fig. 2(a). The material of the lens was specified as glass with a refractive index of 1.51 at a wavelength of $800 \mathrm{~nm}$. The central position of the lens is located at the origin of the global coordinate system, as shown in Fig. 2(a). The overlap area of the spherical geometries has a refractive index of 1.51, which is the same as that of the lens, so that the light entering the lens can travel without any boundary effects (i.e., refraction and/or reflection) in that area.

Two optical photon sources (point sources) were located at the left side of the lens with an object distance of $30 \mathrm{~mm}$ ( $Z=-30 \mathrm{~mm}$ in global coordinates), as shown in Fig. 3(a). The physical size of the point sources was $0 \mathrm{~mm}$ in $X-, Y$-, and $Z$-directions. Thus, the point sources are not visible in (a)

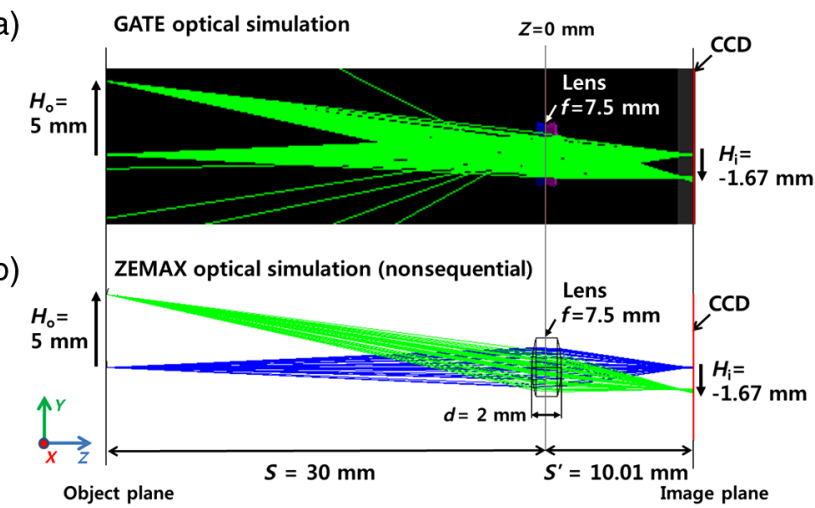

Fig. 3 Comparison of the ray diagrams between (a) the GATE optical simulation and (b) the ZEMAX optical simulation (nonsequential mode).

Fig. 3. One optical photon source was placed along the principal axis without offset in the $Y$-direction (global coordinate: $X=0 \mathrm{~mm}, Y=0 \mathrm{~mm}, Z=-30 \mathrm{~mm}$, and $H_{0}=0 \mathrm{~mm}$ ), and the other optical photon source was located at an offset of $5 \mathrm{~mm}$ in the $Y$-direction (global coordinate: $X=0 \mathrm{~mm}$, $Y=5 \mathrm{~mm}, Z=-30 \mathrm{~mm}$, and $H_{0}=5 \mathrm{~mm}$ ), which corresponds to the object height of $5 \mathrm{~mm}$, as shown in Fig. 3(a). As a result, the two optical photon sources were $5 \mathrm{~mm}$ apart in the $Y$-direction. The off-axis angle of the optical photon source (i.e., a 5 -mm offset in the $Y$-direction; $H_{0}=5 \mathrm{~mm}$ ) with respect to the principal axis ( $Z$-axis) was $10 \mathrm{deg}$. To generate NIR photons, the energy of the optical photons was set to $1.5498 \mathrm{eV}$, which corresponded to a wavelength of $800 \mathrm{~nm}$. Each optical photon source had a flux of $10^{4} \# \mathrm{Ph}$./s. The optical photons were irradiated onto the lens with a cone angle of $3 \mathrm{deg}$ for $1 \mathrm{~s}$ with a total photon incidence rate of $2 \times 10^{4} \mathrm{\# Ph}$./s. The NIR wavelength of $800 \mathrm{~nm}$ was chosen for the GATE optical imaging simulation because the NIR penetrates relatively deeper in biological tissue than the bioluminescence spectral range of 400 to $650 \mathrm{~nm}^{13}$

A charge-coupled device (CCD) detector with dimensions of $10 \mathrm{~mm} \times 10 \mathrm{~mm} \times 1 \mathrm{~mm}$ (width $\times$ height $\times$ depth) was placed at the right side of the lens $(Z=+10 \mathrm{~mm})$ as shown in Fig. 3(a). The material of the CCD detector was defined as silicon (red) as shown in Fig. 3(a). To create an optical interface on the front side of the CCD, an air box volume (gray) with dimensions of $10 \mathrm{~mm} \times 10 \mathrm{~mm} \times 1 \mathrm{~mm}$ (width $\times$ height $\times$ depth) was placed in front of the CCD detector. Then, the surface type of the optical interface between the air box and the CCD was set (a) Biconvex lens implemented in GATE

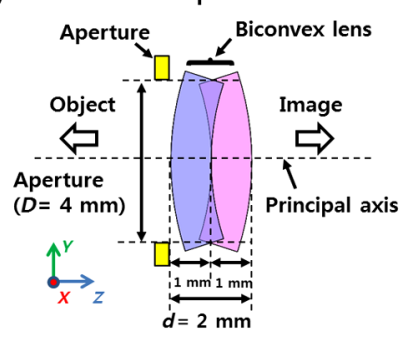

(b) Spherical geometry design

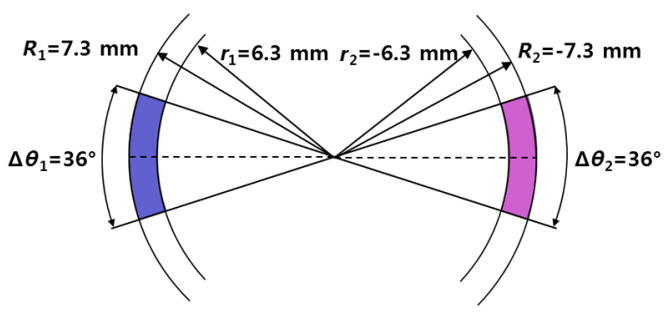

Fig. 2 Schematic of the lens design: (a) the biconvex lens consisting of two spherical geometries used in the GATE optical imaging simulation and (b) the spherical geometry design. 
to "dielectric_metal" to detect optical photons. ${ }^{10}$ The surface finish and surface roughness $\left(\sigma_{\alpha}\right)$ of the optical interface were set to "polished" and $0 \mathrm{deg}$, respectively. In GATE simulation, the matrix size of the CCD detector was $256 \times 256$ $(X \times Y)$, and the physical pixel size was $392 \mu \mathrm{m} \times 392 \mu \mathrm{m}$ in $X$ - and $Y$-directions, respectively. The quantum efficiency of the CCD was set to $100 \%$ from 200 to $900 \mathrm{~nm}$.

\subsection{Validation of the GATE Lens Using ZEMAX Software}

To validate the lens implemented on GATE, a ZEMAX optical simulation was used. The identical geometries of the lens, optical photon sources, and CCD detector were defined in ZEMAX using the nonsequential component editor. The object type and material of the lens were defined as a "standard lens" and "BK7," respectively. The refractive index of the "BK7" was 1.51 at a wavelength of $800 \mathrm{~nm}$, and the diameter of the lens was set to $4 \mathrm{~mm}$ as well as the GATE simulation.

The object types of the two optical photon sources were defined as "source point," and the wavelengths of the sources were set to $800 \mathrm{~nm}(1.5498 \mathrm{eV})$. The two optical point sources were assumed to be sizeless as well as in the GATE simulation setup. The two optical photon sources were placed at the left side of the lens with an object distance of $30 \mathrm{~mm}$ as shown in Fig. 3(b). The number of analysis rays and the power of the each optical photon source was set to $10^{4}$ and $2.479 \times 10^{-15} \mathrm{~J} / \mathrm{s}$, respectively, which resulted in a total photon incidence rate of $2 \times 10^{4} \mathrm{\# Ph}$./s.

To define a CCD detector, the object type of "detector rectangle," material of "ABSORB" was used, respectively. The CCD detector has dimensions of $10 \mathrm{~mm} \times 10 \mathrm{~mm}$ (width $\times$ height). The matrix size of the CCD detector was set to $256 \times 256(X \times Y)$, which resulted in a physical pixel size of $392 \mu \mathrm{m} \times 392 \mu \mathrm{m}$ in $X$ - and $Y$-directions, respectively.

\subsection{Comparison of the CCD Image Between the GATE and ZEMAX}

The CCD image of the GATE was obtained by importing the GATE output file called "Hits.dat," which contains the information such as the photon interaction position with the CCD in the $X$-, $Y$-, and $Z$-directions, and the deposited photon energy.
The GATE output file was imported using MATLAB (R2015a, MathWorks) and a two-dimensional (2-D) CCD image with a matrix size of $256 \times 256(X \times Y)$ was generated. The units of the CCD image were the number of photons per pixel.

The CCD image of the ZEMAX simulation was obtained from the detector viewer result (incoherent irradiance), which contained the deposited energy for each pixel in units of $\mathrm{W} / \mathrm{cm}^{2}$. The Fresnel reflection was taken into account in both the ZEMAX and GATE simulations by selecting the "use polarization" option. The detector viewer result was imported into MATLAB and a 2-D CCD image with a matrix size of $256 \times 256(X \times Y)$ was generated. The pixel units were converted from $\mathrm{W} / \mathrm{cm}^{2}$ to the number of photons per pixel (the pixel size was $392 \mu \mathrm{m} \times 392 \mu \mathrm{m}$ ). The physical CCD pixel size $(392 \mu \mathrm{m} \times 392 \mu \mathrm{m})$ was converted to the object pixel size $(118 \mu \mathrm{m} \times 118 \mu \mathrm{m})$ using a magnification factor of -0.33 so that the CCD image represented the actual object size. Unless otherwise specified, all of the CCD images of GATE and ZEMAX represent the actual object size. To compare the PSF, the line profile of the CCD image was obtained along the $Y$-direction, the pixel value of the line profile was averaged across the $X$-direction from -5 to $+5 \mathrm{~mm}$, and the PSF was calculated based on the full-width at half maximum (FWHM) of a Gaussian fitting of the line profile.

\subsection{Comparison of Resolving Power Between the GATE and ZEMAX Using a USAF 1951 Resolution Target}

To validate the resolving power of the GATE against ZEMAX, a USAF 1951 resolution target source was implemented in both GATE and ZEMAX optical simulations as shown in Figs. 4(a) and $4(\mathrm{~b})$.

The USAF 1951 resolution target consists of the bar patterns represented by the group number 0 and 1 , which of each has six elements. Each element consists of vertical and horizontal patterns, each of which has three bars as shown in Fig. 4(b). The three bars are separated by the spaces of equal width. The width of the largest bar in the group number 0 and element number 1 [hereafter, $(\mathrm{G} 0, \mathrm{E} 1)$ ] is $0.5 \mathrm{~mm}$ as shown in Fig. 4(b). The bar width is decreased with a ratio of $1: 2^{-1 / 6}$ as the element number is increased. The height of the bar is five times long as its width. The optical photon flux and the wavelength of the USAF 1951 (a)

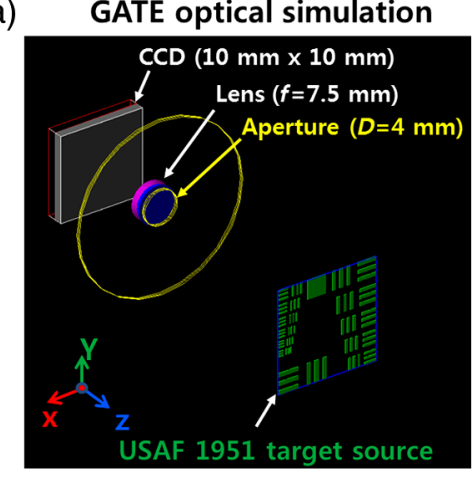

(b)
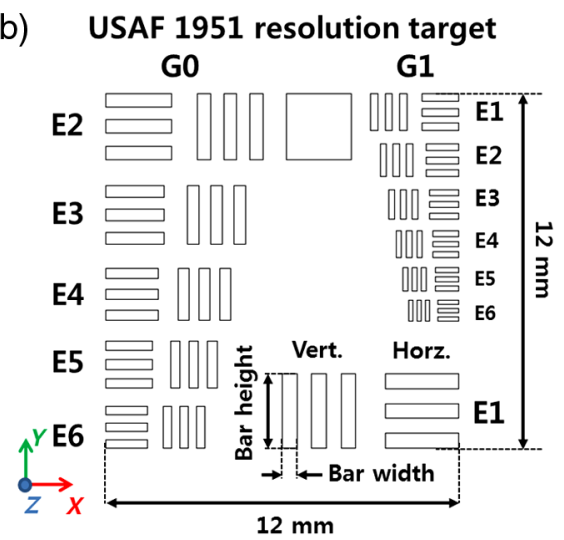

Fig. 4 (a) GATE optical simulation of a USAF 1951 resolution target and (b) USAF 1951 resolution target implemented in ZEMAX optical simulation. Note that the element number 1 (E1) of the group number $O(G 0)$ is located at the right and bottom region of the USAF 1951 resolution target. 
resolution target source were set to $1 \times 10^{6} \# \mathrm{Ph} . / \mathrm{s} / \mathrm{mm}^{2}$ and $800 \mathrm{~nm}$, respectively. The total area of the USAF resolution target source was about $39.04 \mathrm{~mm}^{2}$. The CCD image of the USAF 1951 resolution target was obtained for $60 \mathrm{~s}$ with an object distance of $30 \mathrm{~mm}$. The line profile of each element was obtained in vertical and horizontal directions for the evaluation of contrast. The contrast transfer function (CTF) of each element was calculated using Eq. (5) as follows: ${ }^{14}$

Contrast $(f)=\frac{I_{\max }-I_{\min }}{I_{\max }+I_{\min }}$,

where $I_{\max }$ and $I_{\min }$ are, respectively, the maximum and minimum pixel intensities inside the region of the three bars, and $f$ is the spatial frequency defined as the number of bars per millimeter (line pairs $/ \mathrm{mm}$ ). The $I_{\max }$ was calculated by averaging the pixel intensities of three peaks in the line profile, and $I_{\text {min }}$ was calculated by averaging the pixel intensities of two valleys in the same line profile. The modulus of the CTF was plotted against the spatial frequency.

\subsection{Comparison of CCD Image Between Lens and Pinhole Using GATE}

To demonstrate the advantage of the lens over the pinhole in terms of optical imaging quality, the CCD images of a USAF 1951 resolution target were compared between the biconvex lens and pinhole using the GATE optical simulation. The USAF 1951 resolution target image was obtained with different pinhole diameters $(0.3$, and $0.1 \mathrm{~mm})$ without a biconvex lens. The optical photon flux and wavelength of the optical photon were set to $1 \times 10^{6} \mathrm{Hh} . / \mathrm{s} / \mathrm{mm}^{2}$ and $800 \mathrm{~nm}$, respectively, which were identical to the case of the biconvex lens simulation.

\subsection{Bioluminescence Imaging Simulation Using GATE}

A bioluminescence imaging simulation was performed using the GATE to investigate the effect of optical phantom on the image quality. The optical system described in the previous section was used. The distance between the central position of the lens and the optical photon sources was $30 \mathrm{~mm}$, as shown in Fig. 5(a). An aperture that was $4 \mathrm{~mm}$ in diameter was placed in front of the lens to prevent the stray light from reaching the CCD detector. NIR optical photons with a wavelength of $800 \mathrm{~nm}$ $(=1.5498 \mathrm{eV})$ were emitted from the two cylindrical sources,
Table 1 Optical properties of the various phantoms. ${ }^{10,15}$

\begin{tabular}{lccccc} 
Phantom & $\begin{array}{c}\text { Refractive } \\
\text { index }\end{array}$ & $\mu_{a}\left(\mathrm{~cm}^{-1}\right)$ & $\begin{array}{c}\mu_{s} \\
\left(\mathrm{~cm}^{-1}\right)\end{array}$ & $\begin{array}{c}\mu_{s}^{\prime} \\
\left(\mathrm{cm}^{-1}\right)\end{array}$ & Anisotropy $(\mathrm{g})$ \\
\hline $\begin{array}{l}\text { Scattering } \\
\text { material }\end{array}$ & 1.33 & $2.0 \times 10^{-5}$ & 0.05 & 0.02 & 0.6 \\
Hypodermis & 1.37 & $1.3 \times 10^{-3}$ & 0.63 & 0.126 & 0.8 \\
Epidermis & 1.37 & $3.3 \times 10^{-3}$ & 1.37 & 0.274 & 0.8 \\
\hline
\end{tabular}

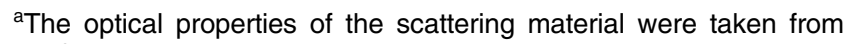
Ref. 10.

which had a diameter of $1 \mathrm{~mm}$ and height of $5 \mathrm{~mm}$, in an isotropic manner with the total optical photon flux of $2 \times 10^{7}$ \#Ph./s as shown in Figs. 5(b) and 5(c). A bioluminescence image was acquired for $1 \mathrm{~s}$ using various phantoms, such as scattering material, hypodermis, and epidermis. ${ }^{10,15}$ The reduced Mie scattering coefficient $\mu_{s}^{\prime}$ was calculated as follows:

$\mu_{s}^{\prime}=\mu_{s}(1-g)$,

where $\mu_{s}$ is the Mie scattering coefficient and $g$ is the Mie anisotropy of an optical scattering material, respectively. The Mie forward anisotropy and backward anisotropies were set to be identical each other. The Rayleigh scattering coefficient of the scattering material was set to $9.26 \times 10^{-3}\left(\mathrm{~cm}^{-1}\right)$, which corresponds to a Rayleigh scattering length of $10.8 \mathrm{~mm}$. The optical parameters of the various phantoms are summarized in Table 1 . The physical cross section of the phantoms was $40 \mathrm{~mm} \times 40 \mathrm{~mm}$, and bioluminescence images were obtained for phantom depths of $0,5,10$, and $15 \mathrm{~mm}$, respectively. The quantum efficiency of the CCD was set to $100 \%$ for the wavelengths from 200 to $900 \mathrm{~nm}$ as described in Sec. 2.2. Unless otherwise specified, the CCD was assumed to be perfect. All simulations were performed using a quad core Intel CPU (2.4 GHz) processor with $4 \mathrm{~GB}$ RAM.

\subsection{Fluorescence Imaging Simulation Using GATE With ICG Fluorophore}

Fluorescence imaging was simulated using the GATE with ICG fluorophore to explore the feasibility of NIR fluorescence imaging as shown in Fig. 6(a). The ICG fluorophore with
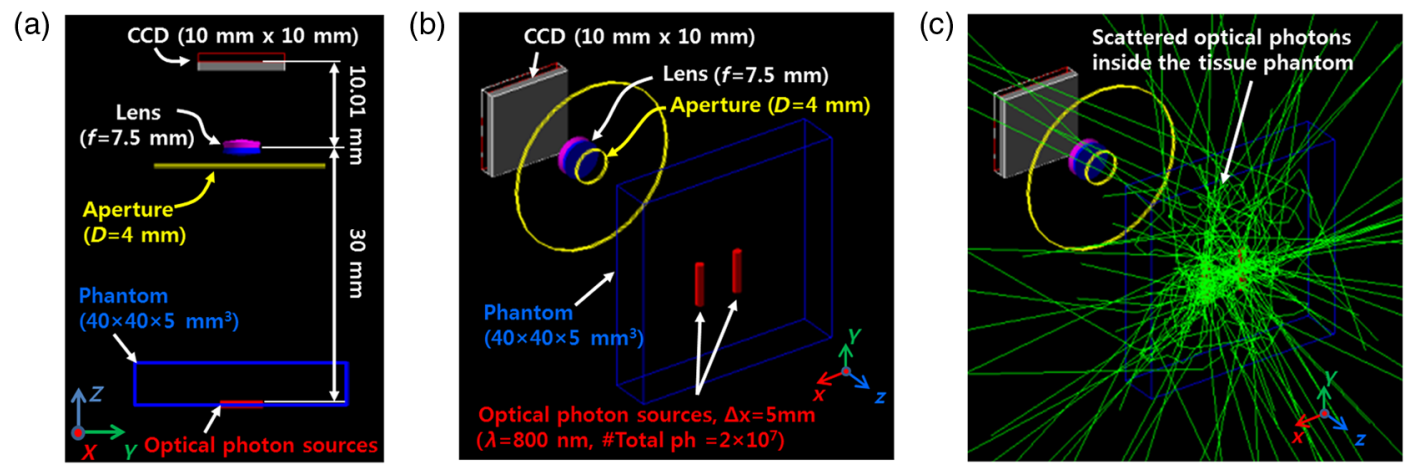

Fig. 5 GATE bioluminescence imaging simulation setup: (a) side view, (b) isotropic view, and (c) scattered optical photons inside the hypodermis tissue phantom with a $5 \mathrm{~mm}$ in depth. 
(a) Fluorescence imaging (ICG)

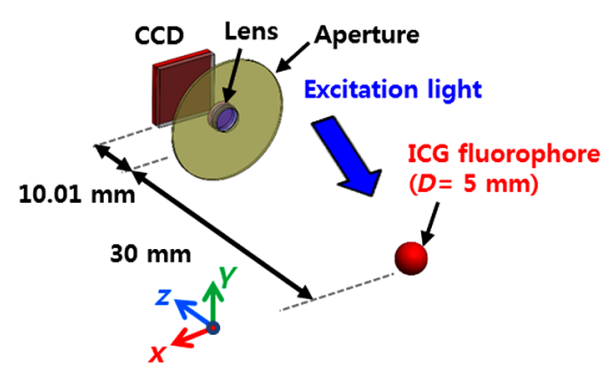

(b) Absorption and emission spectra (ICG)

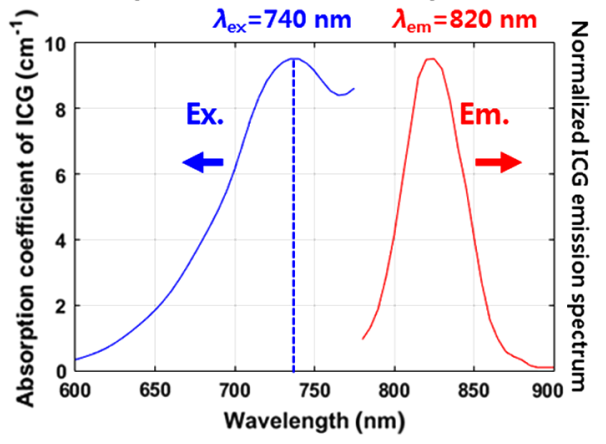

Fig. 6 (a) GATE fluorescence imaging simulation setup and (b) absorption and emission spectra of ICG fluorophore for the fluorescence imaging simulation.

a diameter of $5 \mathrm{~mm}$ was placed $30 \mathrm{~mm}$ away from the central position of the lens. The molar extinction coefficient of ICG in plasma ${ }^{16}$ was converted to an absorption coefficient by multiplying it with the molar concentration of ICG $(100 \mu \mathrm{M})$ using the following equation:

$\mu_{a}=\varepsilon c$,

where $\mu_{a}$ is the absorption coefficient of ICG $\left(\mathrm{cm}^{-1}\right), \varepsilon$ is the molar extinction coefficient of ICG $\left(\mathrm{cm}^{-1} \cdot \mathrm{M}\right)$, and $c$ is the molar concentration of ICG $\left(\mathrm{cm}^{-1} \cdot \mathrm{M}\right)$. The emission spectrum of the ICG was adopted from ISS data. ${ }^{17,18}$ The absorption and emission spectra were resampled with a wavelength interval of $5 \mathrm{~nm}$, and their wavelengths were set to 740 and $820 \mathrm{~nm}$, respectively, as shown in Fig. 6(b). The fluorescence lifetime $\tau$ was set to $27.5 \mathrm{~ns},{ }^{19}$ and the peak fluorescence quantum yield of the ICG was set to 0.084 at a peak emission wavelength of $820 \mathrm{~nm}^{20}$ The excitation light $(740 \mathrm{~nm})$ was irradiated into the ICG fluorophore $(D=5 \mathrm{~mm})$ with a photon flux of $10^{8}$ photons/s for $1 \mathrm{~s}$. The incidence angle of the excitation light with respect to the principal axis was $30 \mathrm{deg}$, and the fluorescence image was obtained with different depths of scattering phantom $(0,5$, and $10 \mathrm{~mm})$. The reflected excitation light on the surface of the scattering phantom was removed and only the fluorescence emission light ranging from 780 to $900 \mathrm{~nm}$ was taken into account for the fluorescence image generation.

\section{Results}

\subsection{Validation of GATE Lens Using ZEMAX Software}

To validate the lens implemented on the GATE, the CCD image of the GATE was compared with that obtained from the ZEMAX. Figures 7(a) and 7(b) show the CCD images obtained with GATE optical simulation and ZEMAX optical simulation (nonsequential mode), respectively. For the visibility of the two point sources (apart $5 \mathrm{~mm}$ in $Y$-direction each other) of each $\mathrm{CCD}$ image, the region of interest (width $=2.5 \mathrm{~mm}$, height $=$ $6.5 \mathrm{~mm}$ ) was cropped and enlarged as shown in the insets in Figs. 7(a) and 7(b). In the case of the GATE, the central positions of the two point sources were 0.0013 and $4.8925 \mathrm{~mm}$, respectively. In the case of ZEMAX, the central positions of the two point sources were 0.0001 and $4.8820 \mathrm{~mm}$, respectively, as shown in Table 2. The positional differences between the GATE and ZEMAX were 1.22 and $10.48 \mu \mathrm{m}$ for the central source and 5-mm off-axis source, respectively. The coma aberration of the lens caused by the 5-mm off-axis point source can be found in both the GATE and ZEMAX results as shown in Fig. 7. Figure 8 shows the line profiles obtained along the $Y$-direction. Subsequently, Gaussian fitting was performed for each peak to calculate the FWHM, and the FWHMs of the central point source were 0.2184 and $0.1919 \mathrm{~mm}$ for the GATE and (a) GATE optical simulation

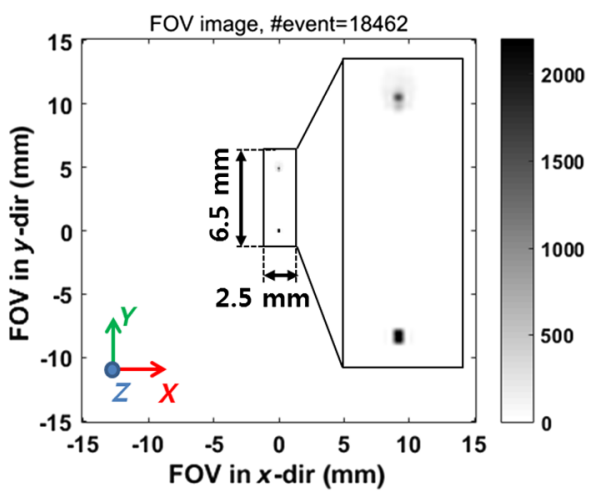

(b) ZEMAX (non-sequential)

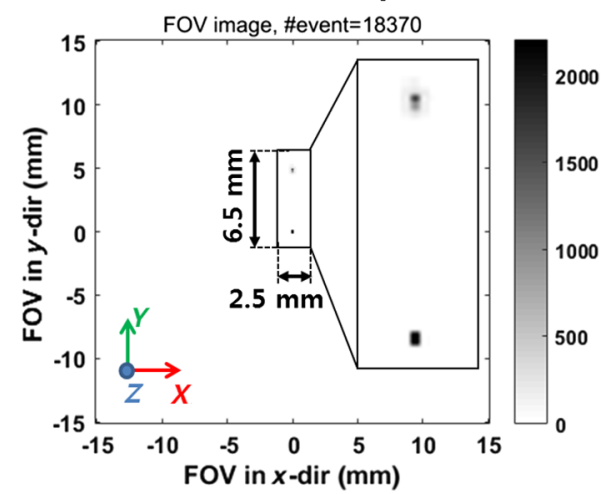

Fig. 7 Comparison of the CCD images between (a) the GATE optical simulation and (b) the ZEMAX simulation (nonsequential mode). (The ROI with a size of $2.5 \mathrm{~mm} \times 6.5 \mathrm{~mm}$ was enlarged as shown in the insets.) 
Table 2 Comparison of the CCD images between the GATE and ZEMAX.

\begin{tabular}{llcc} 
& & & \\
& $\begin{array}{c}\text { Point source position in } \\
\text { the } Y \text {-direction }(\mathrm{mm})\end{array}$ & 0 & 5 \\
\hline GATE & Position $(\mathrm{mm})$ & 0.0013 & 4.8925 \\
& FWHM $(\mathrm{mm})$ & 0.2184 & 0.3785 \\
ZEMAX & Position $(\mathrm{mm})$ & 0.0001 & 4.8820 \\
& FWHM $(\mathrm{mm})$ & 0.1919 & 0.3811 \\
Difference & & & \\
& Position difference $(\mu \mathrm{m})$ & 1.22 & 10.48 \\
& FWHM difference $(\mu \mathrm{m})$ & 26.52 & -2.63 \\
\hline${ }^{a}$ Difference between the GATE and ZEMAX results in units of $\mu \mathrm{m}$.
\end{tabular}

(a) GATE optical simulation

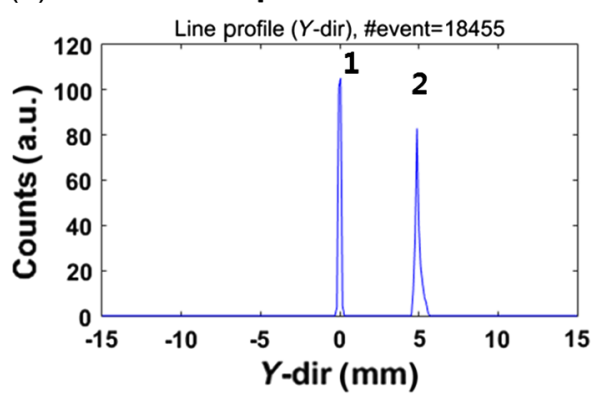

ZEMAX, respectively. The FWHMs of the offset point source (5-mm offset in the $Y$-direction) were 0.3785 and $0.3811 \mathrm{~mm}$ for the GATE and ZEMAX, respectively, as shown in Table 2. The numbers of photons detected on the CCD were 18,462 and 18,370 for GATE and ZEMAX, respectively. The light losses in the interfaces between the lens and air were $8.37 \%$ and $8.87 \%$ for the GATE and ZEMAX, respectively.

\subsection{Comparison of USAF 1951 Resolution Target Images Between GATE and ZEMAX}

The USAF 1951 resolution target images of the GATE and ZEMAX were obtained as shown in Fig. 9. The number of detected photons were $2,391,125 ; 1,953,895 ; 1,545,380$ for GATE and 2,422,949; 1,960,857; and 1,549,226 for ZEMAX with aperture diameters of $4.0,3.6$, and $3.2 \mathrm{~mm}$, respectively.

Fig. 8 Comparison of the line profiles along the $Y$-direction between (a) the GATE optical simulation and

(b) the ZEMAX simulation (nonsequential mode).
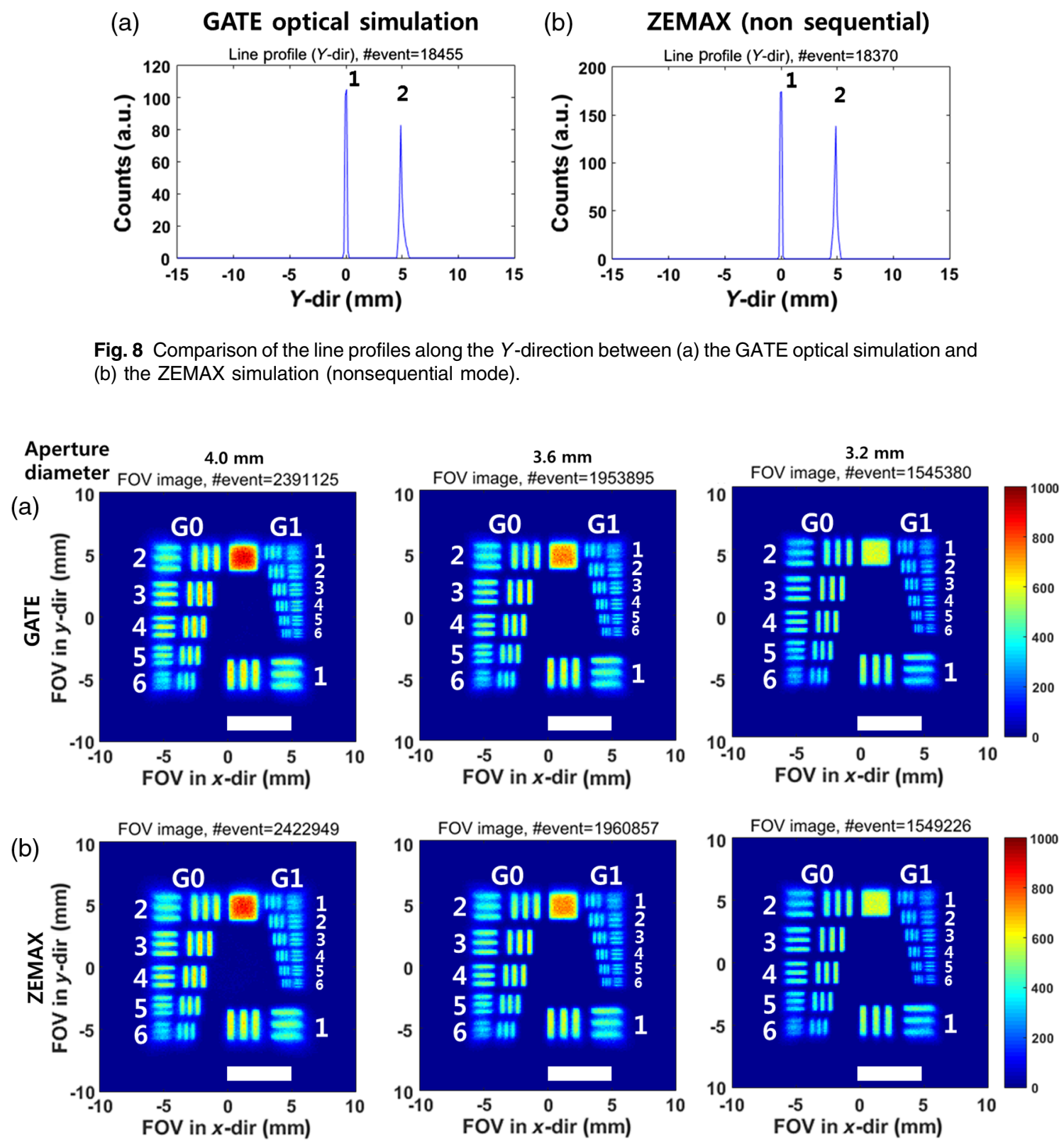

Fig. 9 Comparison of the USAF 1951 resolution target images between (a) the GATE optical simulation and (b) the ZEMAX simulation (nonsequential mode). White horizontal scale bar length $=5 \mathrm{~mm}$. 

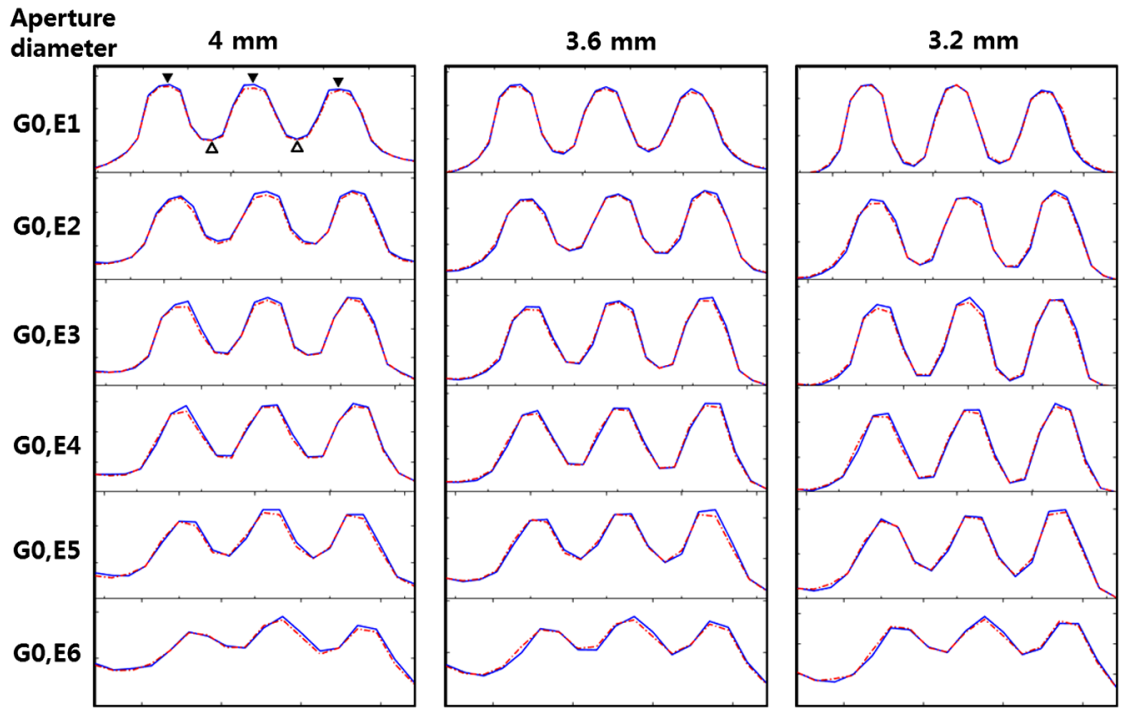

Fig. 10 Comparison of the line profiles of vertical bars in (G0, E1 to E6) between GATE (blue solid line) and ZEMAX (red dotted line) as a function of the aperture diameter (4.0, 3.6, and $3.2 \mathrm{~mm}$ ).

The sensitivities were $6.13 \%, 5.01 \%, 3.96 \%$ for GATE and $6.21 \%, 5.03 \%, 3.97 \%$ for ZEMAX, respectively.

The line profiles of each vertical and horizontal elements were obtained and the pixel intensities of the three peaks (black arrow heads) and two valleys (white arrow heads) were extracted for the calculation of CTF in each element as shown in Fig. 10. The line profiles of the vertical and horizontal patterns (group number 0, element number 1 to 6 ) obtained with GATE showed a good agreement with those of the ZEMAX as shown in Figs. 10 and 11. The bar patterns in G1 were obviously more difficult to resolve than those in G0 as shown in Figs. 12 and 13.

Although the use of smaller aperture reduced the sensitivity, the spatial resolution of the optical systems was improved for both GATE and ZEMAX since the coma aberration was minimized as shown in Figs. 14-16. The CTFs of the GATE depending on the aperture diameter agreed well with those of the ZEMAX as shown in Figs. 15-17.

\subsection{Comparison of USAF 1951 Resolution Target Images Between Pinhole Optics and Lens System}

The USAF 1951 resolution target images of the biconvex lens and pinhole were obtained as shown in Fig. 17(a). The number of detected photons was 11,787 and 861 for the pinhole apertures of 0.3 and $0.1 \mathrm{~mm}$, respectively. The sensitivities of the pinhole apertures of 0.3 and $0.1 \mathrm{~mm}$ were $0.03 \%$ and $0.002 \%$, respectively. The line profile of the vertical bar patterns in $(\mathrm{G} 0, \mathrm{E} 1)$ was obtained with the region of interest (ROI) drawn by a white rectangle as shown in Fig. 17(b). With the pinhole aperture of $0.3 \mathrm{~mm}$, the three vertical bars in $(\mathrm{G} 0, \mathrm{E} 1)$ were hardly resolved as shown in Fig. 17(b). However, the three vertical bars could be resolved as the pinhole aperture of $0.1 \mathrm{~mm}$ was used as shown in Fig. 17(b). The smaller pinhole aperture of $0.1 \mathrm{~mm}$ could improve the spatial resolution as compared with the pinhole aperture of $0.3 \mathrm{~mm}$ as shown in Fig. 17. On the other hand, the sensitivity must be sacrificed considerably because the
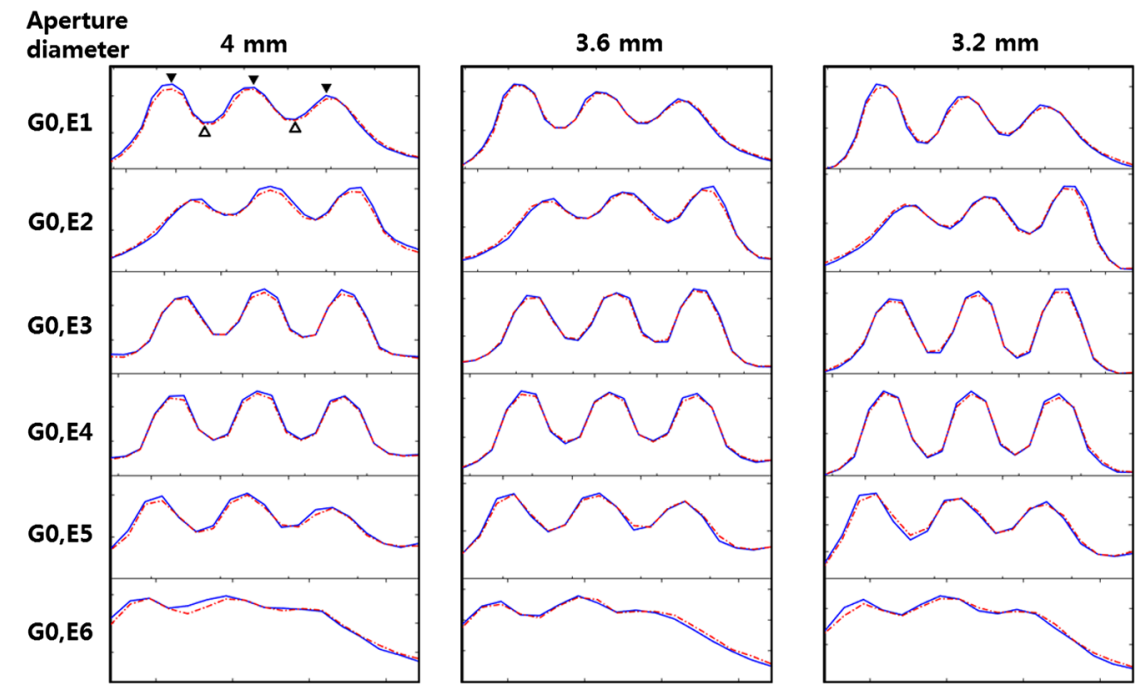

Fig. 11 Comparison of the line profiles of horizontal bars in (G0, E1 to E6) between GATE (blue solid line) and ZEMAX (red dotted line) as a function of the aperture diameter (4.0, 3.6, and $3.2 \mathrm{~mm}$ ). 

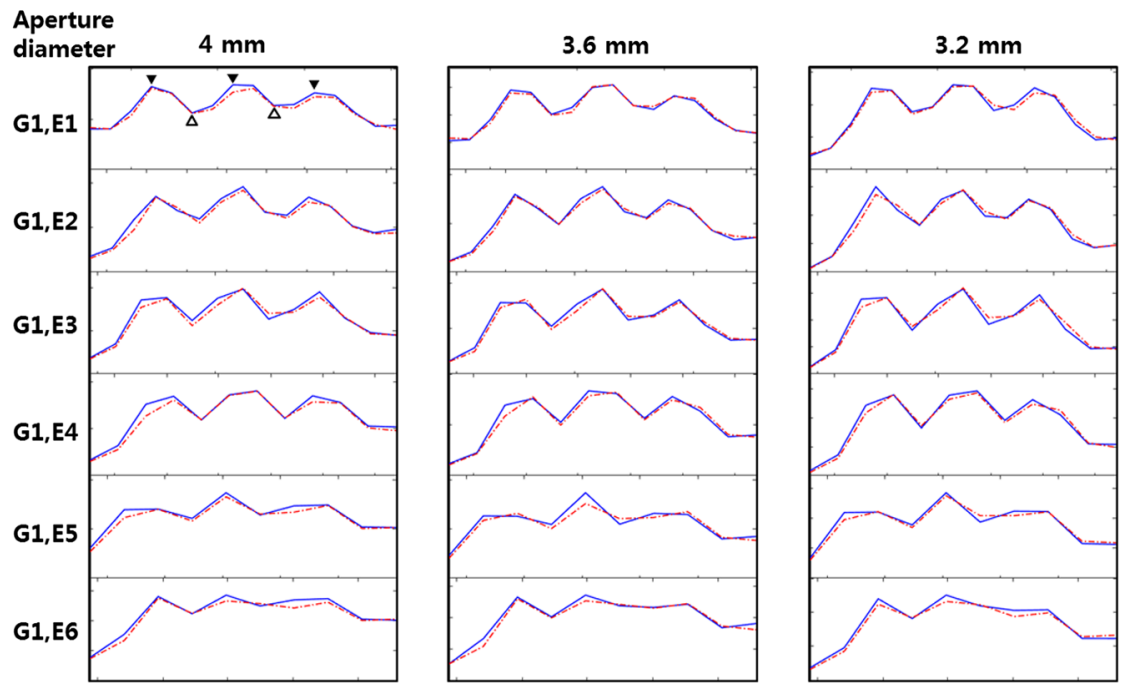

Fig. 12 Comparison of the line profiles of vertical bars in (G1, E1 to E6) between GATE (blue solid line) and ZEMAX (red dotted line) as a function of the aperture diameter (4.0, 3.6, and $3.2 \mathrm{~mm}$ ).
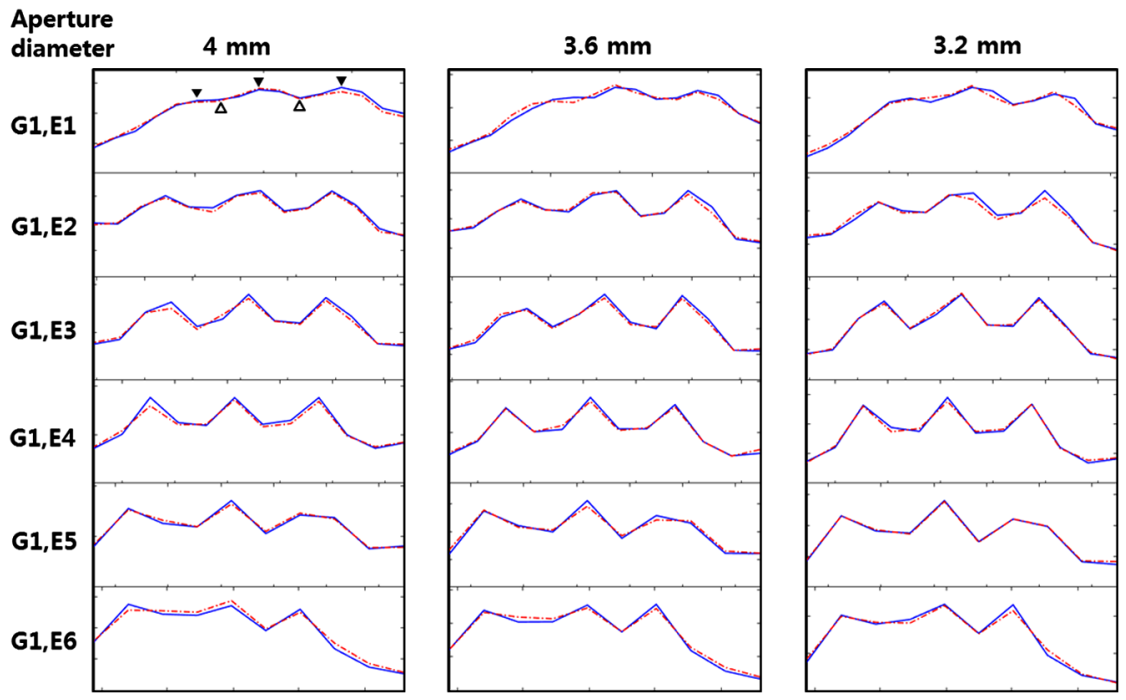

Fig. 13 Comparison of the line profiles of horizontal bars in (G1, E1 to E6) between GATE (blue solid line) and ZEMAX (red dotted line) as a function of the aperture diameter (4.0, 3.6, and $3.2 \mathrm{~mm}$ ).
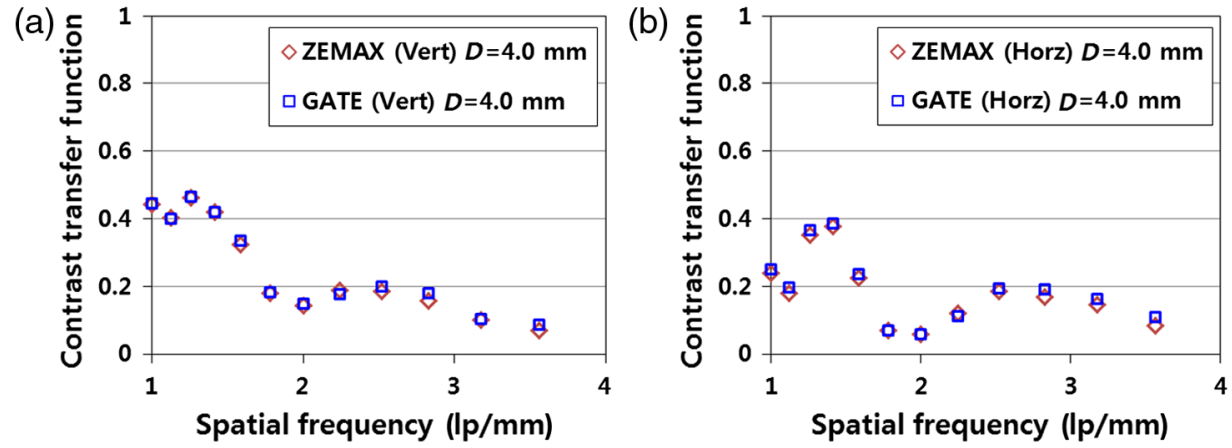

Fig. 14 Comparison of the CTFs between GATE (blue square) and ZEMAX (red diamond) with an aperture diameter of $4 \mathrm{~mm}$ : (a) vertical CTF and (b) horizontal CTF. 
(a)

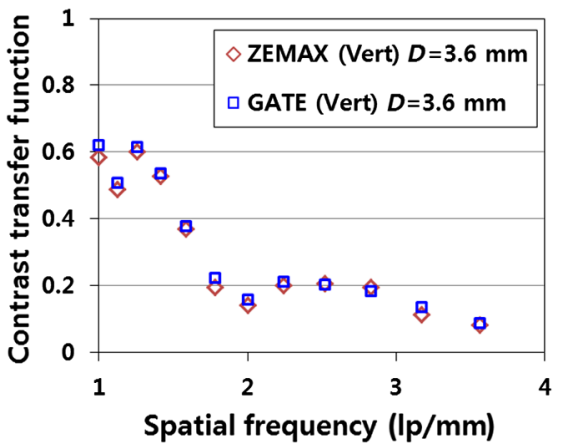

(b)

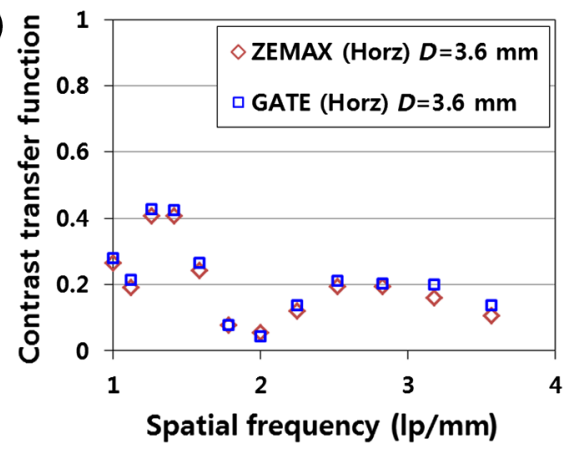

Fig. 15 Comparison of the CTFs between GATE (blue square) and ZEMAX (red diamond) with an aperture diameter of $3.6 \mathrm{~mm}$ : (a) vertical CTF and (b) horizontal CTF.

(a)

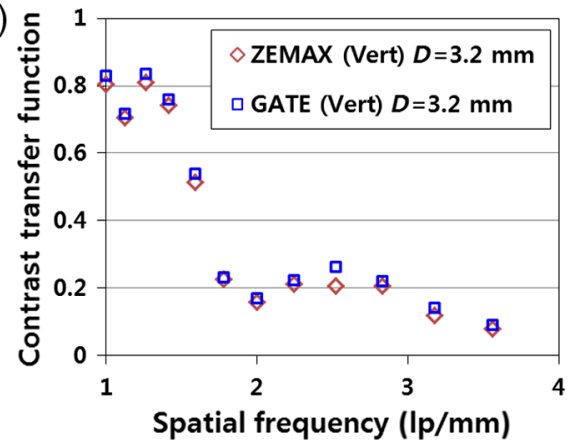

(b)

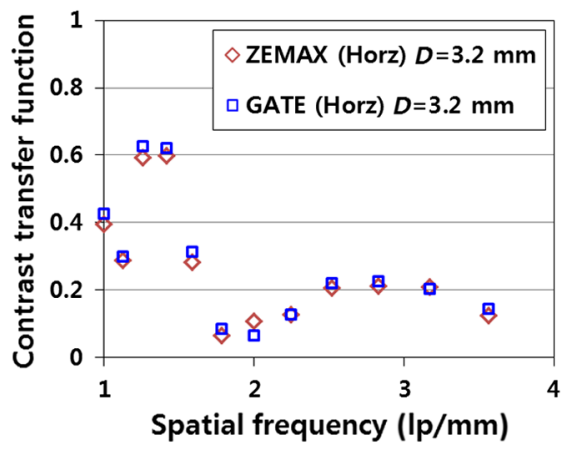

Fig. 16 Comparison of the CTFs between GATE (blue square) and ZEMAX (red diamond) with an aperture diameter of $3.2 \mathrm{~mm}$ : (a) vertical CTF and (b) horizontal CTF.

Lens

(Aperture $=3.2 \mathrm{~mm}$ )

(a)

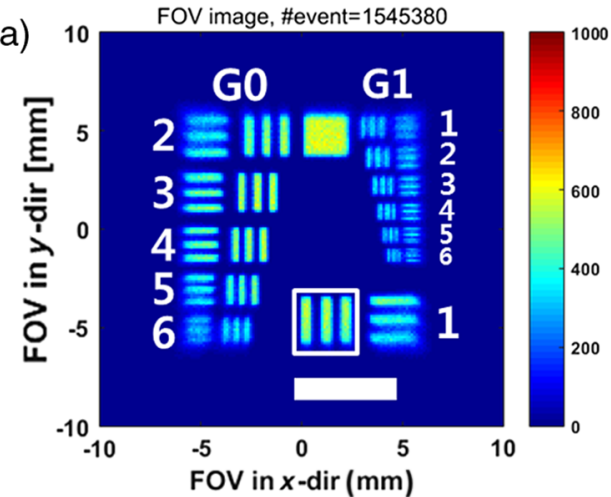

(b)

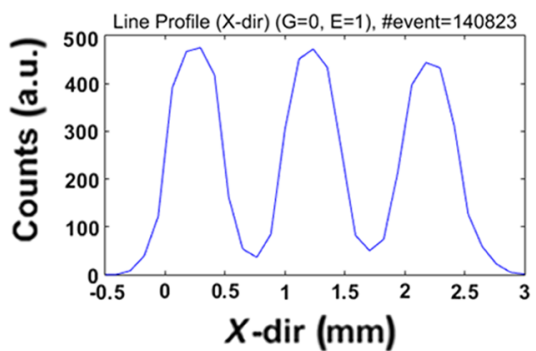

Pinhole

(Aperture $=0.3 \mathrm{~mm}$ )

FOV image, \#event $=11787$
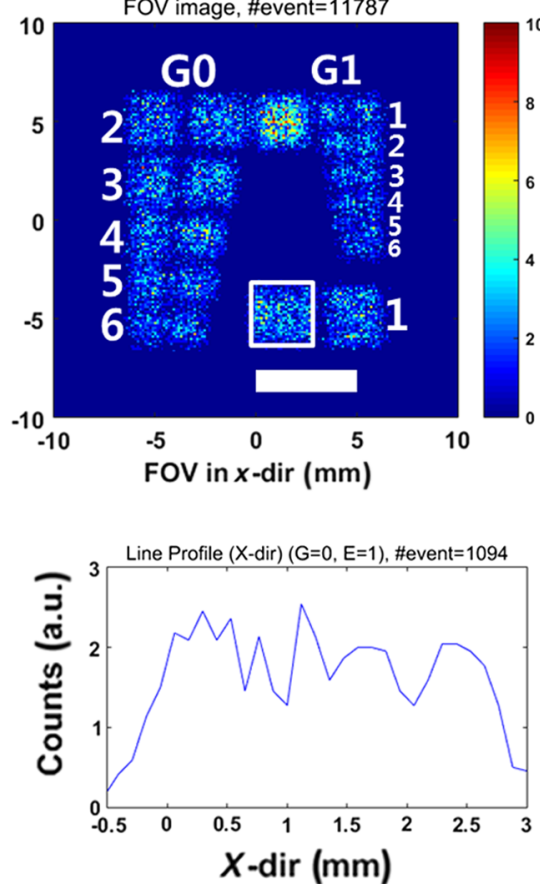

Pinhole

(Aperture $=0.1 \mathrm{~mm}$ )

FOV image, \#event $=861$
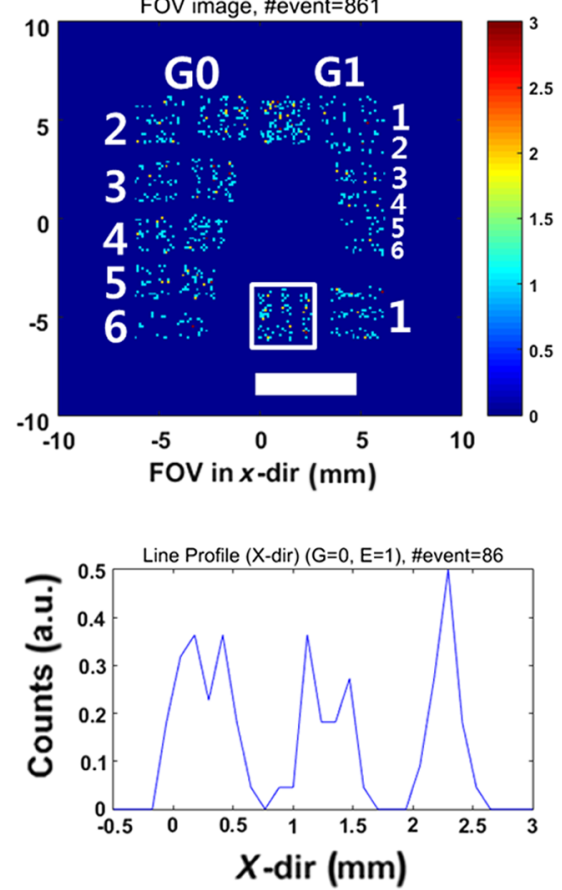

Fig. 17 Comparison of the USAF 1951 resolution target images between the biconvex lens and pinhole optics: (a) CCD image and (b) line profile of the vertical bars in (G0, E1) obtained from the white rectangular ROI. White horizontal scale bar length $=5 \mathrm{~mm}$. 
sensitivity is proportional to the area of the pinhole aperture. However, the sensitivity could be improved substantially from $0.03 \%$ to $3.96 \%$ using the biconvex lens as compared with the pinhole optics (aperture $=0.3 \mathrm{~mm}$ ) without compromising the spatial resolution.

\subsection{Bioluminescence Imaging Using the GATE With Various Phantoms}

The bioluminescence image of two cylindrical sources was simulated with different depths of various optical phantoms $(0,5$, and $10 \mathrm{~mm})$. In the case of the CCD image without the optical phantom, the cylindrical source with 5-mm offset in the $X$-direction was blurry compared with the cylindrical source located at the center of the object plane due to the coma aberration, as shown in Fig. 18. The two cylindrical sources could be distinguished in the scattering phantom of 5-mm depth. However, the two cylindrical sources were barely resolved with the scattering phantom of $10-\mathrm{mm}$ depth as shown in Fig. 18. In the case of the hypodermis phantom, the two cylindrical sources could not be distinguished even for a phantom depth of $5 \mathrm{~mm}$, as shown in Fig. 19. In the case of the epidermis phantom, the light absorption was more severe than for the hypodermis phantom since the epidermis had a higher absorption coefficient $\left(3.3 \times 10^{-3} \mathrm{~cm}^{-1}\right)$, than the hypodermis phantom $\left(1.3 \times 10^{-3} \mathrm{~cm}^{-1}\right)$, as shown in Fig. 20 . The number of detected optical photons was calculated from the ROI marked by white dotted rectangle as shown in Figs. 18-20. The relative number of optical photons detected on the CCD was significantly affected by both the depth and type of the optical phantom, as shown in Fig. 21. The effect of phantom depth on the light attenuation was more severe as the optical absorption coefficient was increased as shown in Fig. 21.

\subsection{Fluorescence Imaging Simulation Using GATE}

Fluorescence imaging was simulated using an ICG NIR fluorophore sphere ( $D=5 \mathrm{~mm}$ ) using the setup described in Fig. 6(a). The fluorescence images of the ICG fluorophore sphere with different scattering phantom depths are shown in Fig. 22(a). The line profile of the NIR fluorescence image along the $X$-direction was obtained with a 1-mm thickness in the $Y$-direction, as shown in Fig. 22(b). The distance between the two falling edges of the line profile was consistent with the physical size of the ICG fluorophore $(D=5 \mathrm{~mm})$. Figure 22(c) shows the NIR fluorescence emission spectrum detected on the CCD, which agrees with the ICG emission spectrum defined in Sec. 2.8. The number of detected fluorescence emission photons was 122,$939 ; 23,776$; and 4183 for scattering phantom depths of 0,5 , and $10 \mathrm{~mm}$, respectively.

\section{Discussion}

In this paper, we demonstrated the feasibility of several optical imaging simulation techniques, namely bioluminescence and NIR fluorescence imaging, with the GATE MC toolkit combined with our implementation of a biconvex lens. The lens implementation in the GATE was validated using the ZEMAX software, which is an established optical simulation software package, as shown in Fig. 3. The maximum discrepancies in the position and FWHM were $10.48 \mu \mathrm{m}$ and $26.52 \mu \mathrm{m}$, respectively, between the GATE and ZEMAX, as shown in Table 2. The maximum discrepancy in the position $(10.48 \mu \mathrm{m})$ between GATE and ZEMAX was also smaller than the CCD object pixel size $(118 \mu \mathrm{m})$, which indicates a good agreement between GATE and ZEMAX in terms of the CCD image. When 200,000 optical photons at a cone angle of $3 \mathrm{deg}$ were irradiated into the lens, as shown in Fig. 3, the number of detected photons on the CCD was 18,455 and 18,370 in the GATE and ZEMAX
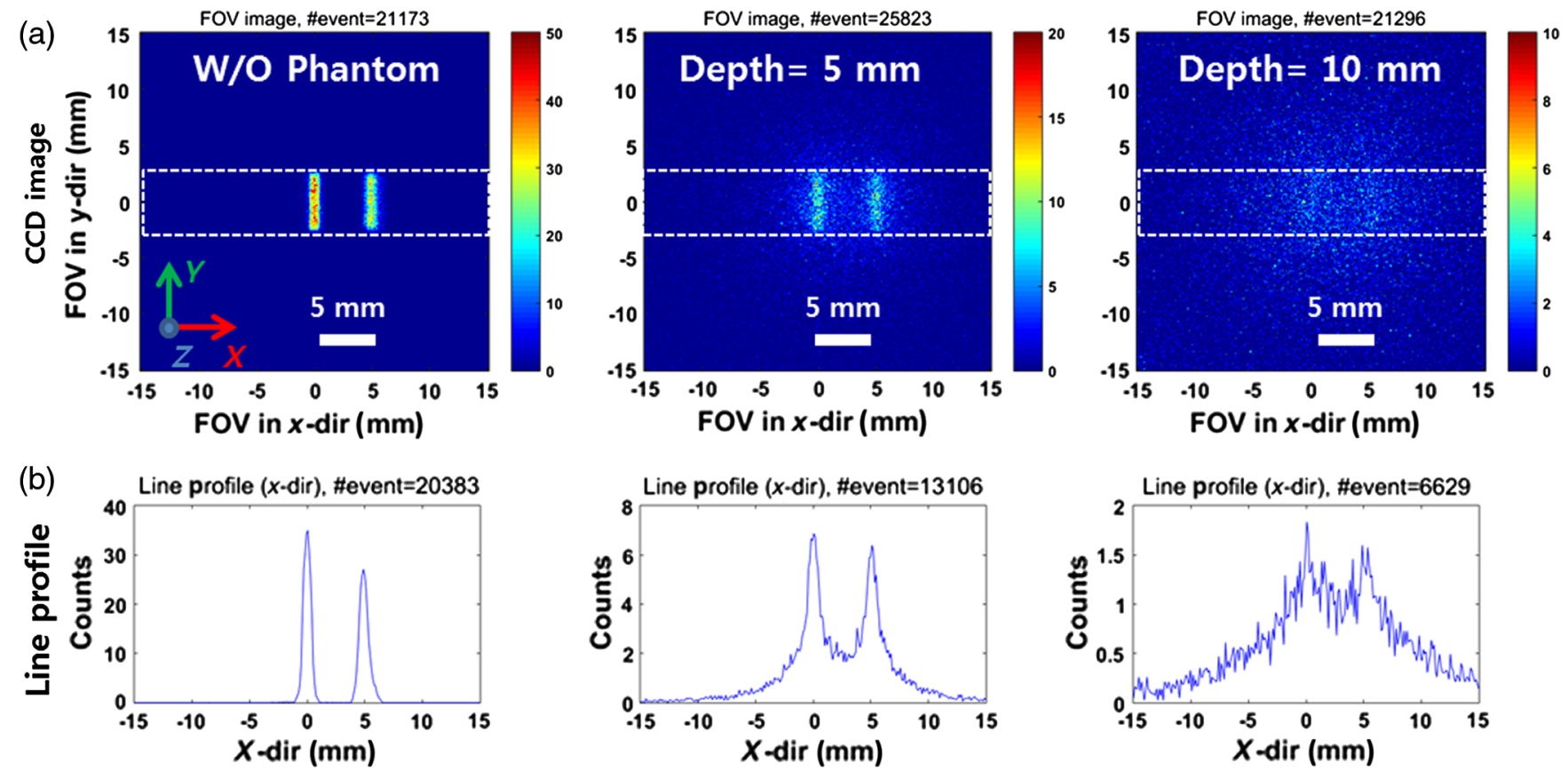

Fig. 18 GATE bioluminescence imaging results with different scattering phantom depths: (a) CCD image and (b) line profiles along the $X$-direction. 

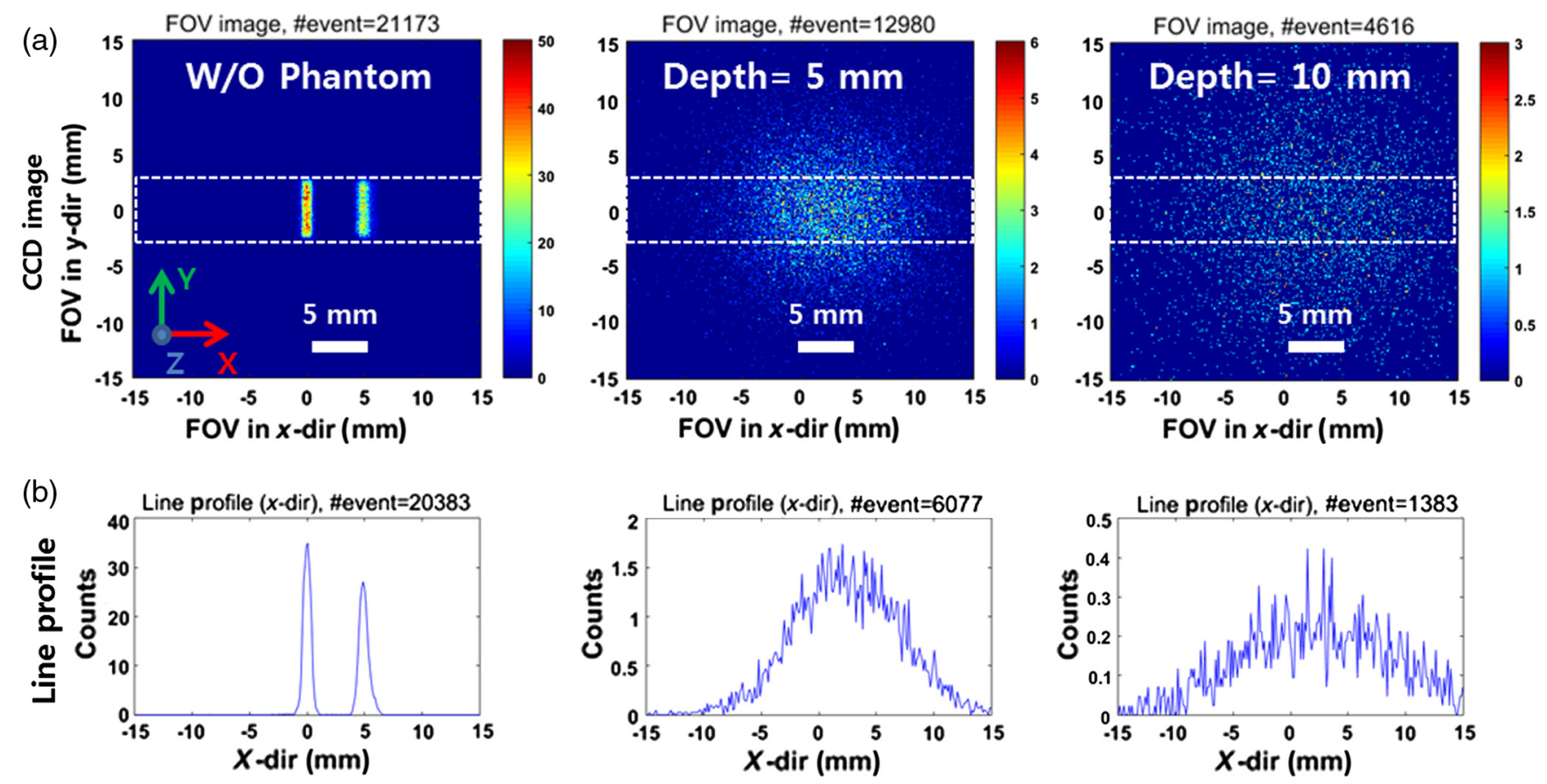

Fig. 19 GATE bioluminescence imaging results with different hypodermis phantom depths: (a) CCD image and (b) line profiles along the $X$-direction.

simulations, respectively, as shown in Fig. 8. The observed light losses of $8.37 \%$ and $8.87 \%$ for the GATE and ZEMAX simulations, respectively, were caused by the Fresnel reflection that occurred in the boundary of the biconvex lens. ${ }^{21}$ The percent difference of the optical photon counts between the GATE and ZEMAX simulations was only $0.46 \%$.

The CCD images of the USAF 1951 resolution target obtained with the GATE optical simulation showed a good agreement with those of obtained with the ZEMAX in terms of the CTF as shown in Figs. 9 and 14. These results confirm that the biconvex lens implementation in the GATE was successful. In the CCD image of the USAF 1951 resolution target, the vertical bar patterns in (G0, E1 to E6) could be resolved clearly as shown in Fig. 10. However, the horizontal bar pattern (group number 0, element number 6) located at the left bottom corner of the USAF 1951 resolution target was barely resolved
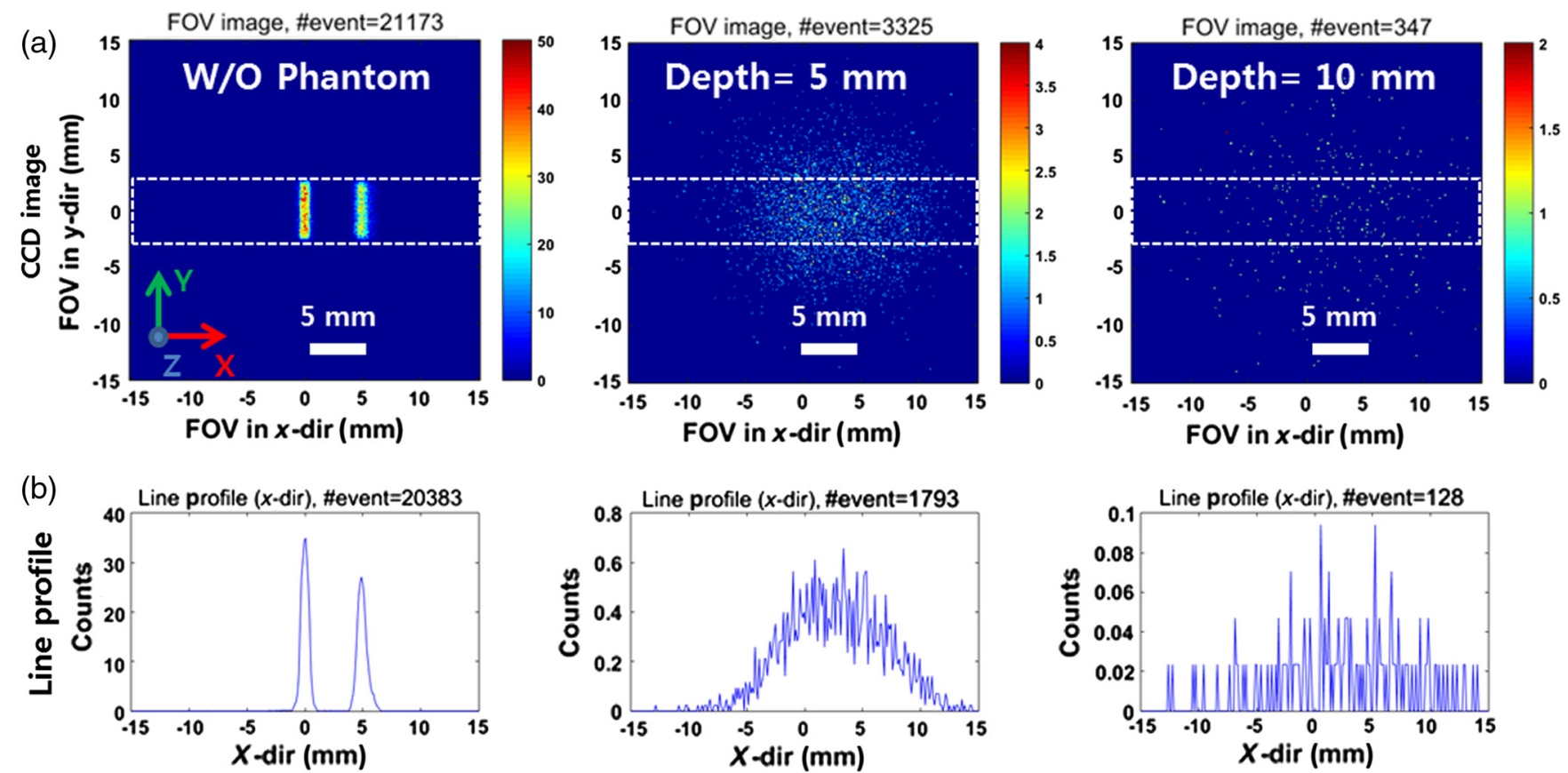

Fig. 20 GATE bioluminescence imaging results with different epidermis phantom depths: (a) CCD image and (b) line profiles along the $X$-direction. 


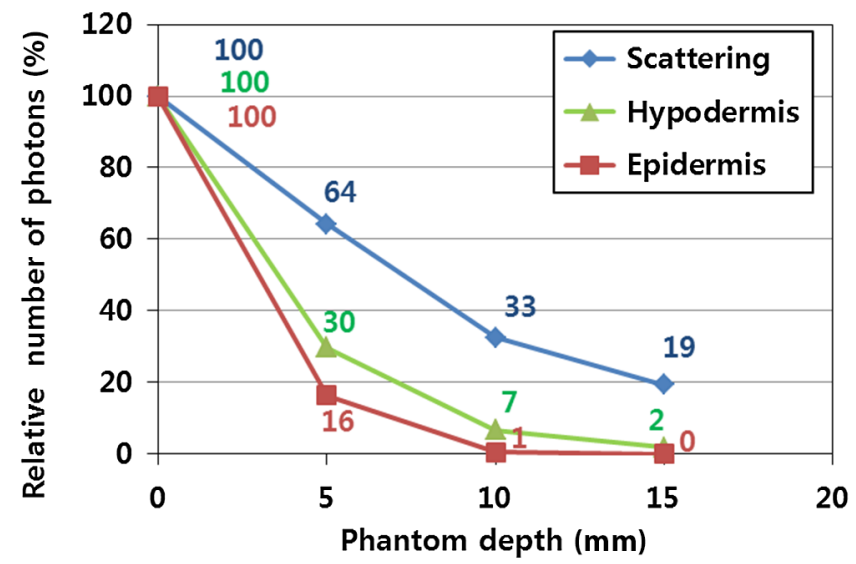

Fig. 21 Relative number of optical photons detected on the CCD as a function of the phantom depth with various materials (GATE bioluminescence imaging simulation).

due to the coma aberration as shown in Figs. 9 and 11. The vertical bars pattern in (G0, E4) could be resolved, which corresponds to a spatial resolution of $2.83(\mathrm{lp} / \mathrm{mm})$ as shown in Fig. 12. Unlike the vertical bar pattern of (G1, E1), the horizontal bar pattern of (G1, E1) located at the right upper corner of the USAF 1951 resolution target was hardly resolved due to the coma aberration as shown in Figs. 9 and 13.

The CTF of the lens system could be improved using a smaller aperture diameter since the coma aberration can be minimized as shown in Figs. 14-16. However, the sensitivity was degraded significantly due to the reduced aperture diameter. The CTF obtained with the USAF 1951 resolution target was affected by the location of the bar pattern on the image plane as shown in Figs. 14-16. This is mainly because of the coma aberration, which resulted in a position dependent the PSF of the lens on the image plane as shown in Fig. 7.

The sensitivities of the biconvex lens (aperture diameter $=$ $3.2 \mathrm{~mm}$ ) and pinhole optics (aperture diameter $=0.3 \mathrm{~mm}$ ) were $3.96 \%$ and $0.03 \%$, respectively. Unlike the biconvex lens, the pinhole optics with an aperture diameter of $0.3 \mathrm{~mm}$ could not resolve the vertical bar pattern $(\mathrm{G} 0, \mathrm{E} 1)$ as shown in Fig. 17. Although, the vertical bar pattern could be distinguished as the pinhole aperture diameter was decreased from 0.3 to $0.1 \mathrm{~mm}$, the sensitivity was degraded substantially from $0.03 \%$ to $0.002 \%$. Unlike the pinhole optics, which was used by Helo et al. for the simulation of Cerenkov imaging-based quality assurance of electron radiotherapy, ${ }^{22}$ the use of a lens can (a) Fluorescence image (ICG)
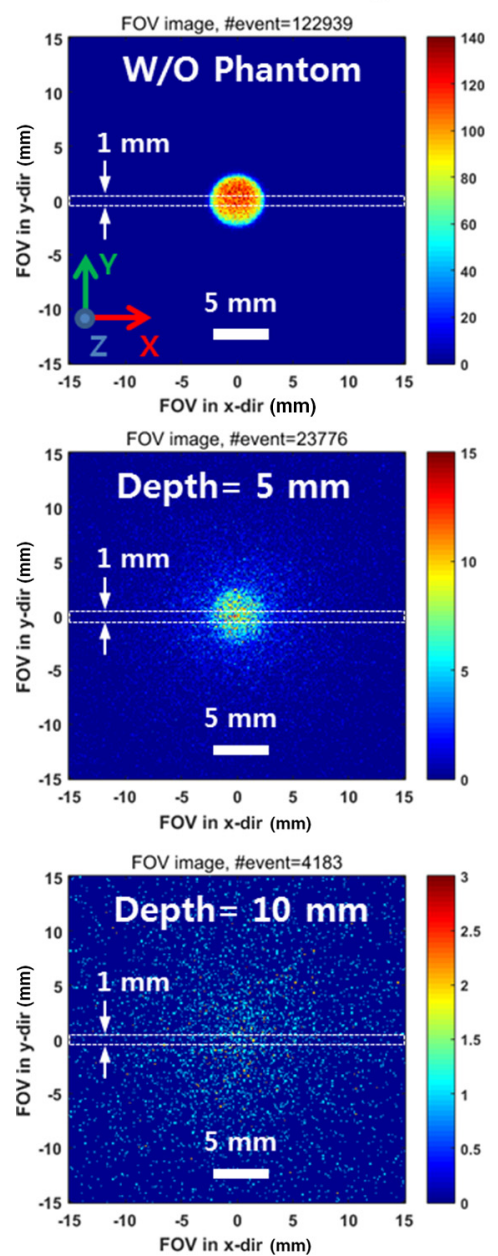

(b) Line profile (X-dir)
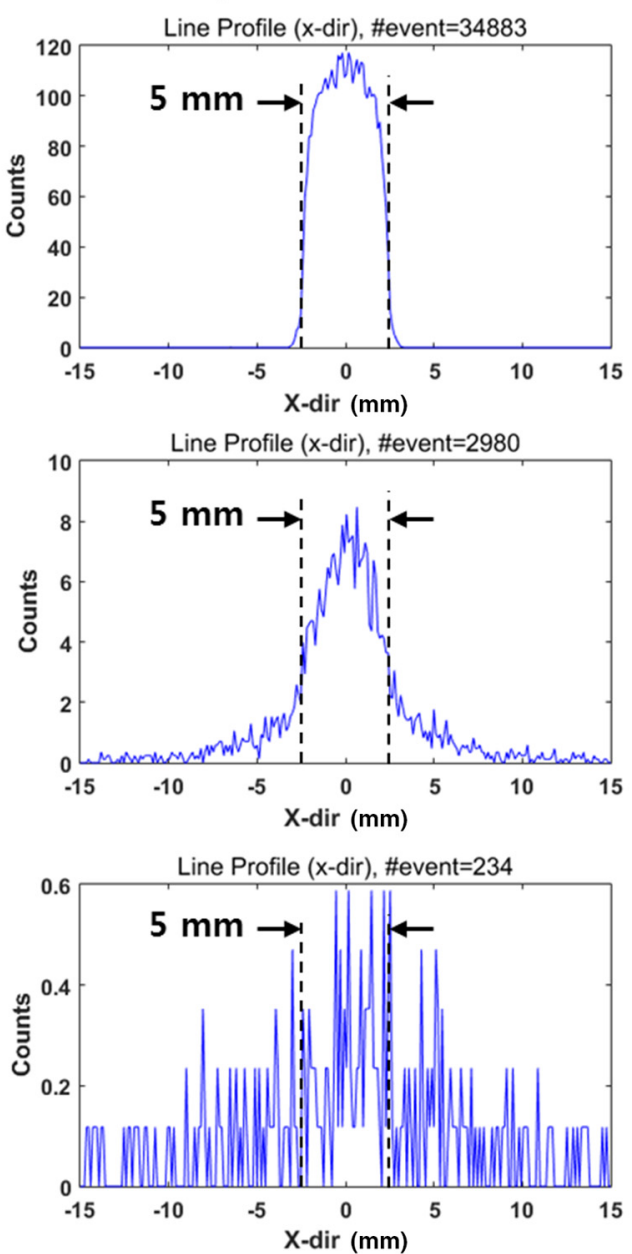

(c) Emission spectrum (ICG)
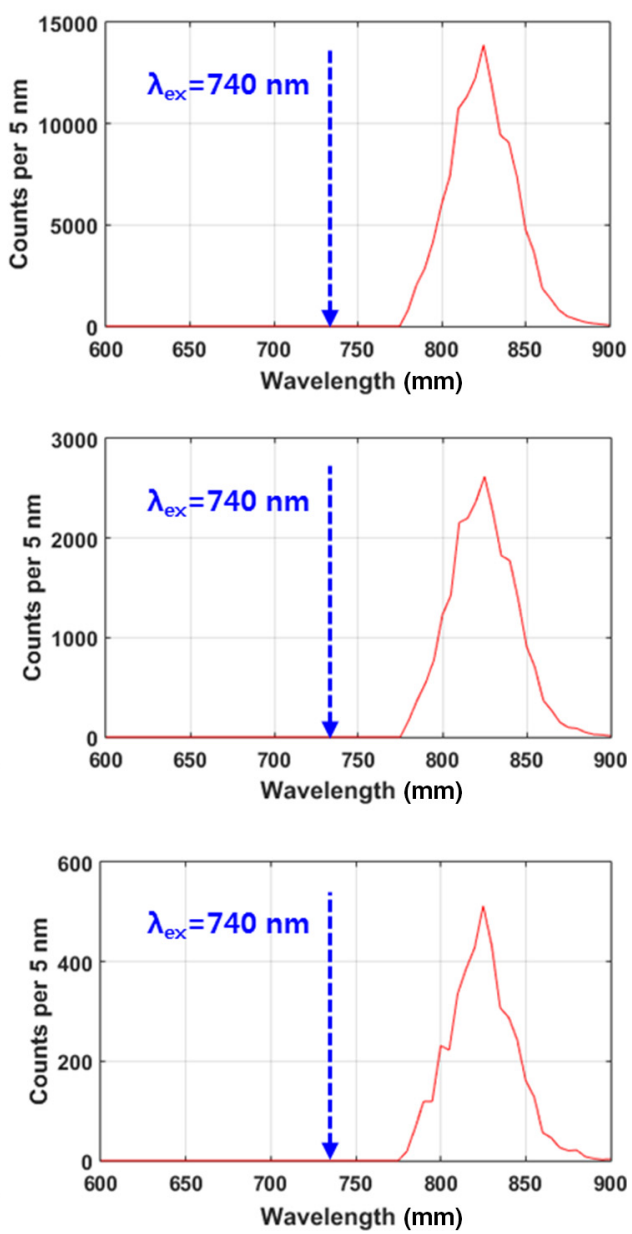

Fig. 22 NIR fluorescence imaging simulation results using ICG fluorophore with different scattering phantom depths: (a) the CCD image, (b) line profile along the $X$-direction, and (c) emission spectrum of the CCD. 
significantly increase the light collection efficiency and spatial resolution in GATE optical imaging simulations as shown in Fig. 17.

In the case of the bioluminescence imaging simulation with various phantoms, the number of detected photons on the CCD was significantly decreased by the optical phantom depth and optical properties of the phantoms, as shown in Fig. 21.

The fluorescence imaging simulation using ICG NIR fluorophore can be performed successfully using the GATE as shown in Fig. 22. The emission spectrum of the ICG agreed well with the predefined emission spectrum. Moreover, the excitation and emission spectra of other fluorophores such as IRDye $800 \mathrm{CW}^{23}$ can be adopted into the GATE. The simulation time for the fluorescence imaging was $4 \mathrm{~h} 14 \mathrm{~m}, 4 \mathrm{~h} 34 \mathrm{~m}$, and $5 \mathrm{~h} 56 \mathrm{~m}$ for the scattering phantom depths of 0,5 , and $10 \mathrm{~mm}$, respectively. Although the number of excitation photons $\left(10^{8}\right)$ was same for each scattering depth, the simulation time was increased due to the increased optical paths with the phantom depth. ${ }^{10}$

One of the significant advantages of GATE MC simulation toolkit over other optical simulation MC simulation software is that optical/nuclear hybrid imaging modality can be modeled ${ }^{24}$ since the GATE has the capability to simulate both optical photon and ionizing radiation. In the future, we plan to model an IVIS optical imaging system ${ }^{1}$ by employing the detailed specifications of the imaging lens, illumination system, and the CCD.

\section{Conclusion}

We implemented a biconvex lens into GATE MC simulation for various optical imaging simulations such as bioluminescence and fluorescence imaging. The lens implemented into GATE was validated against ZEMAX optical simulation using the USAF 1951 resolution target successfully. The GATE MC simulation toolkit can now be used as a valuable tool for the modeling of various optical imaging systems.

\section{Disclosures}

The authors declare that they have no competing interests.

\section{Acknowledgments}

This work was supported by grants from the Korea Evaluation Institute of Industrial Technology funded by the Ministry of Trade, Industry, and Energy of the Korean Government (2015-4-10051988) and the National Research Foundation (NRF) of Korea of the Ministry of Science, ICT, and Future Planning Nuclear R\&D Program (NRF-2016M2A2A4A03913619).

\section{References}

1. B. W. Rice, M. D. Cable, and M. B. Nelson, "In vivo imaging of lightemitting probes," J. Biomed. Opt. 6(4), 432 (2001).

2. G. D. Luker and K. E. Luker, "Optical imaging: current applications and future directions," J. Nucl. Med. 49(1), 1-4 (2007).

3. H. Liu et al., "Molecular optical imaging with radioactive probes," PLoS One 5(3), e9470 (2010).

4. L. Wang, S. L. Jacques, and L. Zheng, "MCML—Monte Carlo modeling of light transport in multi-layered tissues," Comput. Methods Programs Biomed. 47(2), 131-146 (1995).
5. $\mathrm{H}$. Li et al., "A mouse optical simulation environment (MOSE) to investigate bioluminescent phenomena in the living mouse with the Monte Carlo method," Acad. Radiol. 11(9), 1029-1038 (2004).

6. H. Shen and G. Wang, "A tetrahedron-based inhomogeneous Monte Carlo optical simulator," Phys. Med. Biol. 55(4), 947-962 (2010).

7. G. E. Strangman, Z. Li, and Q. Zhang, "Depth sensitivity and sourcedetector separations for near infrared spectroscopy based on the Colin27 brain template," PLoS One 8(8), e66319 (2013).

8. S. Jan et al., "GATE V6: a major enhancement of the GATE simulation platform enabling modelling of CT and radiotherapy," Phys. Med. Biol. 56(4), 881-901 (2011).

9. S. Jan et al., "GATE: a simulation toolkit for PET and SPECT," Phys. Med. Biol. 49(19), 4543-4561 (2004).

10. V. Cuplov et al., "Extension of the GATE Monte-Carlo simulation package to model bioluminescence and fluorescence imaging," J. Biomed. Opt. 19(2), 026004 (2014).

11. V. Cuplov, F. Pain, and S. Jan, "Simulation of nanoparticle-mediated near-infrared thermal therapy using GATE," Biomed. Opt. Express 8(3), 1665 (2017).

12. M. Young, Optics and Lasers: Including Fibers and Optical Waveguides, Springer-Verlag, Berlin Heidelberg (2000).

13. A. N. Bashkatov et al., "Optical properties of human skin, subcutaneous and mucous tissues in the wavelength range from 400 to $2000 \mathrm{~nm}$," J. Phys. D Appl. Phys. 38(15), 2543-2555 (2005).

14. G. Themelis et al., "Real-time intraoperative fluorescence imaging system using light-absorption correction," J. Biomed. Opt. 14(6), 064012 (2009).

15. C. R. Simpson et al., "Near-infrared optical properties of ex vivo human skin and subcutaneous tissues measured using the Monte Carlo inversion technique," Phys. Med. Biol. 43(9), 2465-2478 (1998).

16. M. L. J. Landsman et al., "Light-absorbing properties, stability, and spectral stabilization of indocyanine green," J. Appl. Physiol. 40(4), 575-583 (1976).

17. "ISS focus and discover (measurement examples)," http://www.iss.com/ resources/research/measurement_examples/index.html.

18. A. V. DSouza et al., "Review of fluorescence guided surgery systems: identification of key performance capabilities beyond indocyanine green imaging," J. Biomed. Opt. 21(8), 080901 (2016).

19. M. Y. Berezin et al., "Near infrared dyes as lifetime solvatochromic probes for micropolarity measurements of biological systems," Biophys. J. 93(8), 2892-2899 (2007).

20. T. J. Russin et al., "Measuring the fluorescent quantum efficiency of indocyanine green encapsulated in nanocomposite particulates," J. Phys. Condens. Matter 22(33), 334217 (2010).

21. S. Zhu et al., "Frustrated total internal reflection: a demonstration and review," Am. J. Phys 54(7), 601-607 (1986).

22. Y. Helo et al., "Imaging Cerenkov emission as a quality assurance tool in electron radiotherapy," Phys. Med. Biol. 59(8), 1963-1978 (2014).

23. F. Wu, M. Tamhane, and M. E. Morris, "Pharmacokinetics, lymph node uptake, and mechanistic PK model of near-infrared dye-labeled bevacizumab after IV and SC administration in mice," AAPS J. 14(2), 252-261 (2012)

24. H. G. Kang et al., "A feasibility study of an integrated NIR/gamma/ visible imaging system for endoscopic sentinel lymph node mapping," Med. Phys. 44(1), 227-239 (2017).

Han Gyu Kang received his BS and MS degrees in radiological science from Eulji University, Seongnam, South Korea, in 2012 and 2014, respectively. He received his $\mathrm{PhD}$ in senior healthcare from Eulji University, Daejeon, South Korea, in 2018. He is the author of more than six journal papers. He holds four patents. His current research interests include the GATE Monte-Carlo simulation for optical imaging and the development of an optical/nuclear multimodal laparoscope for sentinel lymph node mapping. He is a member of SPIE.

Biographies for the other authors are not available. 